\title{
The effect of antihypertensive treatment on arterial stiffness and serum concentration of selected matrix metalloproteinases
}

\author{
Marek Rajzer ${ }^{1}$, Wiktoria Wojciechowska ${ }^{1}$, Tomasz Kameczura², Agnieszka Olszanecka ${ }^{1}$, \\ Danuta Fedak ${ }^{3}$, Michał Terlecki ${ }^{1}$, Kalina Kawecka-Jaszcz ${ }^{1}$, Danuta Czarnecka ${ }^{1}$
}

\begin{abstract}
${ }^{1}$ First Department of Cardiology, Interventional Electrocardiology and Hypertension, Jagiellonian University, Medical College, Krakow, Poland

${ }^{2}$ Chair of Electroradiology, Faculty of Medicine, University of Rzeszow, Rzeszow, Poland ${ }^{3}$ Chair of Clinical Biochemistry and Diagnostics, Department of Clinical Biochemistry, Jagiellonian University Medical College, Krakow, Poland
\end{abstract}

Submitted: 29 July 2015

Accepted: 14 December 2015

Arch Med Sci 2017; 13, 4: 760-770

DOI: https://doi.org/10.5114/aoms.2016.58825

Copyright @ 2016 Termedia \& Banach

\section{Abstract}

Introduction: The aim of the study was to assess the arterial stiffness and serum levels of selected metalloproteinases (MMPs) in hypertensive patients and their changes following antihypertensive therapy.

Material and methods: The study group consisted of 95 patients with essential arterial hypertension (HT) stage 1 or 2 (mean age: $53.1 \pm 13.0$ years). The control group consisted of 31 normotensives of the same age range. Hypertension patients were randomized to one of the following monotherapies for 6 months: quinapril, losartan, amlodipine, hydrochlorothiazide or bisoprolol. Carotid-femoral pulse wave velocity (PWV) was measured using a Complior device. Serum concentrations of MMPs (proMMP-1, MMP-2, MMP-3, MMP-9) and plasma concentration of tissue inhibitor of MMPs (TIMP-1) were measured using ELISA.

Results: Pulse wave velocity and serum concentrations of MMP-2 and MMP-9 were higher in HT patients than in the control group. In HT patients PWV was significantly associated $\left(R^{2}=0.41\right)$ with age $(B=0.408, p=0.00027)$, systolic blood pressure (SBP) $(B=0.441, p=0.0011)$, and MMP-3 $(B=0.204$, $p=0.0459)$. After 6 months of treatment, regardless of the agent used, we observed a significant decrease of PWV, SBP, MMP-2 and MMP-3 and an increase of TIMP-1 plasma concentration. The decrease of PWV was significantly associated with a decrease of SBP $\left(R^{2}=0.07, B=0.260, p=0.015\right)$ only.

Conclusions: In patients with arterial hypertension, beside age and systolic blood pressure, the determinants of arterial stiffness include serum MMP-3 concentration. For drugs compared in the study with the same hypotensive effect obtained, the arterial stiffness reduction effect is not dependent on the drug used. Systolic blood pressure is one of the independent factors responsible for the reduction of arterial stiffness in the course of antihypertensive treatment.

Key words: arterial hypertension, antihypertensive treatment, matrix metalloproteinases, arterial stiffness.

\section{Introduction}

Stiffness of the large arteries is an important factor in the pathogenesis of arterial hypertension and its complications. The most commonly

\author{
Corresponding author: \\ Marek Rajzer \\ First Department \\ of Cardiology Interventional \\ Electrocardiology \\ and Hypertension \\ Jagiellonian University \\ Medical College \\ 17 Kopernika St \\ 31-501 Krakow, Poland \\ Phone: +48 124247300 \\ Fax: +48 124247320 \\ E-mail: rajzer37@interia.pl
}


used method in the assessment of arterial stiffness, carotid-femoral (aortic) pulse wave velocity (PWV), is a strong predictor of survival for the general population, and is not limited to patients with hypertension only $[1,2]$. The 2013 guidelines of the European Society of Hypertension (ESH) and the European Society of Cardiology (ESC) not only recommend the measurement of carotid-femoral PWV in cardiovascular risk stratification in hypertensive patients (class Ila recommendation, level of evidence B) but also emphasize that it is possible to achieve at least partial reversibility of this asymptomatic organ damage following antihypertensive treatment [3]. The reduction of aortic PWV during antihypertensive therapy in patients with end stage renal disease (ESRD) has been associated with an improved survival prognosis [4]. A PWV lowering effect has been previously described for many antihypertensive drugs, primarily for angiotensin-converting enzyme inhibitors and angiotensin receptor blockers [5-9]. According to some authors, the effect of reducing arterial stiffness, which has been described for selected antihypertensive agents, is independent of the lowering of blood pressure by these agents $[10,11]$. Thus, it should be concluded that the effect of reducing arterial stiffness depends on other (non-antihypertensive) properties of certain drugs or classes of drugs.

The development of arterial stiffness is associated with changes in the composition, proportion and physical properties of the structural proteins of the vascular wall, especially collagen and elastin $[12,13]$. The relationship between the plasma concentration of collagen type I and type III metabolites and the stiffness of large arteries under antihypertensive medication with quinapril has been described previously $[14,15]$. Also, a reduction in the plasma concentration of carboxy-terminal collagen type I propeptide (PICP) was observed with use of this drug [8]. Connective tissue matrix metalloproteinases (MMPs) and their tissue inhibitors play a key role in the metabolism of the structural proteins of the vascular wall.

The importance of MMPs in vascular wall remodeling is not limited to their direct effect as proteolytic enzymes in the metabolism of structural proteins. They are also involved in numerous processes such as inflammation, fibrosis, angiogenesis and cell apoptosis [13, 16-18]. Therefore in many diseases with the contribution of a vascular pathology component, e.g. atherosclerosis, plaque complications, various types of vasculitis, diabetes, and chronic kidney disease, the complex role of MMPs is often the subject of research [1924]. In the literature, there are reports on the relationship between the activity of MMPs and their tissue inhibitors and arterial stiffness parameters.
McNulty et al. described a strong positive correlation between plasma levels of MMP-1 (collagenase, mainly responsible for the degradation of type I collagen) and carotid-femoral PWV, and a weak negative correlation between the TIMP-1/ MMP-1 ratio and PWV [14]. For MMP-2 and MMP-9 gelatinases, the effect on vascular remodeling [25], the destruction of elastin laminae and their role in the formation of an aortic aneurysm are described [26]. Also, a relationship between their elevated plasma levels and arterial stiffness [27] was observed.

MMP-3 (stromelysin-1), a metalloproteinase with a very broad spectrum of activities, including the activation of other metalloproteinases such as MMP-1 and MMP-9 (degrading fibrillar collagen and gelatin, respectively), may play an initiating role in the remodeling and stiffening of the walls of large arteries [28]. Overexpression of the MMP-3 gene observed in homozygotes $5 \mathrm{~A}$ of the promoter gene polymorphism $(5 \mathrm{~A} / 6 \mathrm{~A})$ was associated with a higher MMP-3 protein level in the aorta and higher aortic characteristic impedance - an important parameter of aortic stiffness [29]. It may be hypothesized that the effect of antihypertensive drugs on arterial stiffness depends on their impact on MMP activity. Subsequently, a variety of effects on arterial stiffness originate from various effects on metalloproteinases.

There are only a few reports which link a reduction in arterial stiffness during antihypertensive treatment with changes in the activity of metalloproteinases and their inhibitors, and they are mostly based on small groups of patients [30].

Moreover, the available literature data on plasma concentrations of metalloproteinases and their inhibitors in patients with arterial hypertension, as well as data on changes in these parameters after antihypertensive therapy, are often inconsistent and sometimes contradictory [31, 32].

The objective of the study was to assess arterial stiffness and serum levels of selected metalloproteinases in patients with arterial hypertension and changes in the analyzed parameters following treatment with different antihypertensive drugs.

\section{Material and methods}

The study was a scheduled as prospective trial. The study group comprised patients from an outpatient hypertension clinic. Patients with essential arterial hypertension stage 1 and 2 according to the 2013 ESH/ESC Guidelines were enrolled, i.e. arterial blood pressure (BP) values $\geq 140 / 90 \mathrm{~mm} \mathrm{Hg}$ and < 180/110 mm Hg [3], aged 30-75 years, both women and men.

The diagnosis of hypertension, which allowed inclusion in the study, was established based on blood pressure measurements performed twice, 
1 week apart, with BP values as above. The measurements were performed in standard conditions, after 5 min rest, in a sitting position on the non-dominant arm (the mean of the two measurements taken at an interval of $1 \mathrm{~min}$ ).

In patients with previously diagnosed and treated hypertension, these measurements were made after a minimum two-week drug withdrawal period. Enrollment in the study group was completed after reaching the sample size estimated in Power Sample Size analysis considered sufficient to demonstrate a significant difference between the compared treatment groups in: blood pressure parameters, carotid-femoral PWV and plasma levels of MMP-3. Finally, the study group included 95 patients with hypertension (51 men and 44 women). Exclusion criteria for the study were: previous history of coronary artery disease, i.e. myocardial infarction, angina pectoris, a history of coronary revascularization, as well as cardiac valvular disease, atrial fibrillation, prior stroke or transient ischemic attack, antiplatelet, anticoagulant or lipid-lowering therapy, acute or chronic inflammation, cancer, kidney or liver failure. Diabetic patients treated with insulin were also excluded.

A control group of 31 people without hypertension (established by interview and at least two years of medical records of blood pressure measurements with no values $\geq 140 / 90 \mathrm{~mm} \mathrm{Hg}$ ) in the same age range as the study group, with the same proportion of women and men and the same incidence of smoking, was selected on the basis of the same exclusion criteria from patients reporting to periodic preventive checkups at the outpatient clinic.

Diabetes was diagnosed based on the WHO criteria [33]. Obesity was diagnosed based on the value of the body mass index $(\mathrm{BMI}) \geq 30 \mathrm{~kg} / \mathrm{m}^{2}$. Patients smoking one or more cigarettes a day for the past 6 months were considered active smokers.

In the group of hypertensive patients, 57 (60\%) were antihypertensive therapy naïve, while $38 \mathrm{pa}$ tients (40\%) had been previously treated with one or a combination of two antihypertensive drugs at low doses.

In this group 7 patients received previously slow release indapamide $1.5 \mathrm{mg}$ as a monotherapy during the last 1-4 months, 6 patients were treated with lisinopril 5 to $10 \mathrm{mg}$ twice daily as monotherapy for a period of 2-3 months, 6 patients with perindopril $5 \mathrm{mg}$ once daily for a period of 1-3 months, 4 patients with valsartan $80 \mathrm{mg}$ once daily for a period of 1-3 months, 4 patients were treated with verapamil $120 \mathrm{mg} /$ day for $1-3$ months, 3 patients with carvedilol $6.125 \mathrm{mg}$ to $12.5 \mathrm{mg}$ twice daily for a period of 1-4 months, 2 patients with metoprolol succinate $50 \mathrm{mg} /$ day, one for a period of 2 months and the second for
3 months, 6 patients were treated with combination therapy indapamide $0.625 \mathrm{mg}$ plus perindopril $2.5 \mathrm{mg}$ once daily for 2-3 months.

In the diabetic subgroup (6 patients), 3 were treated with metformin 500-850 mg/d before and then during the study period. Other medications during the study (including statins) were prohibited. A low salt and low cholesterol diet was recommended for all participants. In some cases for a short time (no longer than 1 week) nonsteroid anti-infammatory drug (NSAIDs) were used.

Previously administered antihypertensive treatment was discontinued at least 2 weeks before the inclusion of patients in the study. Using a computer-generated randomization list, patients with primary hypertension were allocated to five equal $(n=19)$ groups receiving monotherapy with one of the following five drugs for 6 months: quinapril, amlodipine, hydrochlorothiazide, losartan or bisoprolol. The initial dosage of drugs from the randomization visit were given once a day and were as follows: quinapril $20 \mathrm{mg}$, amlodipine $5 \mathrm{mg}$, hydrochlorothiazide $25 \mathrm{mg}$, losartan $50 \mathrm{mg}$ or bisoprolol $5 \mathrm{mg}$. If the blood pressure goal of less than 140/90 mm Hg was not achieved at the visit after a month of treatment, the dose was doubled. Thus, for five months in the group treated with quinapril, 18 (95\%) patients received a dose of $40 \mathrm{mg}$ once a day, in the group treated with amlodipine, 12 (63\%) patients received one dose of $10 \mathrm{mg}$ a day, in the group of hydrochlorothiazide $12(63 \%)$ patients treated received a dose of $50 \mathrm{mg}$ once a day, 16 (84\%) patients in the group treated with losartan received a dose of $50 \mathrm{mg}$ twice a day and 12 (63\%) patients in the group treated with bisoprolol received a dose of $10 \mathrm{mg}$ once a day. Further control visits were carried out after three and 6 months of treatment. The proportion of patients who achieved a blood pressure reading of $<140 / 90 \mathrm{~mm} \mathrm{Hg}$ at visits after 3 and 6 months did not differ significantly in all therapeutic subgroups, and ranged from 74 to $77 \%$.

\section{Study procedures}

Before any study procedure all subjects signed an informed consent form. The Jagiellonian University Ethics Committee approved the protocol of the study (decision number: KBET/7/B/2007).

During each visit, blood pressure measurements were performed under standard conditions, twice at an interval of one minute on the non-dominant arm, after five minutes rest in the sitting position, in accordance with the recommendations of the European Society of Hypertension [34], using the Omron M5-I oscillometric device (Omron Healthcare Co., Japan) [35]. The average of the obtained values was used for further analyses. Carotid-femoral PWV was measured at 
each visit with a Complior device (Colson, Garges les Genosse, France; software version 2.1) [36]. The PWV measurements were made in accordance with the standards recommended by the "Expert consensus document on arterial stiffness: methodological issues and clinical applications" published in 2006 [1], as well as the "Expert consensus document on the measurement of aortic stiffness in daily practice using carotid-femoral pulse wave velocity" published in 2012 [37]. However, for a more effective comparison of our results with those previously published, the distance measured on the body surface between the carotid and femoral arteries was not multiplied by 0.8 as recommended by the latter document [37]. After overnight fasting, blood was collected from the antecubital vein. Measurements of serum concentrations of glucose, creatinine, total cholesterol, high-density lipoprotein (HDL)-cholesterol, low-density lipoprotein (LDL)-cholesterol and triglycerides were performed. Blood samples for the measurement of serum concentrations of metalloproteinases (proMMP-1, MMP-2, MMP-3 and MMP-9) and TIMP-1 were centrifuged at $3000 \mathrm{~g}$, then the plasma was separated and samples stored at $-75^{\circ} \mathrm{C}$ until analysis. Serum concentrations of metalloproteinases (proMMP-1, MMP-2, MMP-3, MMP-9) and the plasma concentration of TIMP-1 were measured at the initial visit and after 6 months of treatment in all groups. TIMP-1 plasma concentrations were measured using an ELISA kit (Human TIMP-1 Immunoassay, Quantikine, R\&D Systems Europe, Ltd. Abingdon, UK) with an inter-assay variation below $4.9 \%$ and intra-assay variation below $5.0 \%$. The concentration of serum metalloproteinases was measured using kits from R\&D Systems Europe Ltd, Abingdon, UK. Human pro-MMP-1 (inter-assay/intra-assay variation below 5.\%/6.7\%), Human MMP-2 (inter-assay/intra-assay variation below $7.4 \% / 5.3 \%)$, Human MMP-3 (inter-assay/intra-assay variation below 7.9\%/5. 7\%) and Human MMP-9 with inter-assay/ intra-assay variation below $6.9 \% / 2.9 \%$ were used.

\section{Statistical analysis}

Database management and statistical analysis were performed with SAS software (SAS Institute, Cary, NC), version 9.1. The SAS Power and Sample Size application (PSS) was used to estimate sufficient sample sizes to achieve adequate power. Variables were analyzed for normal distribution by the Shapiro-Wilk statistic. The means and proportions were compared using the $t$-test and the $\chi^{2}$ test, respectively. The repeated measures analysis of variance, followed by Tukey's post-hoc tests, was implemented for the comparison of continuous parameters of interest in the study groups. Possible covariates of the changes in PWV during follow-up were investigated using multiple regressions, with the $F$-value for independent variables to enter and stay in the model set at 0.10.

$P$-values $<0.05$ were considered statistically significant.

\section{Results}

Comparison of clinical characteristics of the study and control groups before treatment and at the visit after 6 months is shown in Table I.

Comparison of treatment groups at the first visit (randomization) before treatment and after 6 months of treatment in the study group is shown in Table II.

During the initial visit, the treated and control groups did not differ significantly with respect to age, gender, number of patients with diabetes or smoking before treatment.

Mean systolic and diastolic blood pressure and carotid-femoral PWV were significantly higher in the study group. Plasma concentrations of total cholesterol and LDL-cholesterol and serum concentrations of MMP-2 (231.8 (33.1) vs. 214.2 (42.7), $p=0.022$ ) and MMP-9 (358.1 (165.4) vs. 290.6 (157.7), $p=0.047$ ) were also higher in the study group than in the control group. At the start of the study, the treated and control groups did not differ in other studied concentrations of metalloproteinases and TIMP-1.

In the control group, there were no significant differences in blood pressure, carotid-femoral PWV, plasma concentrations of metalloproteinases or TIMP-1, nor in total cholesterol, LDL or other biochemical parameters between the initial visit and the final visit (after 6 months) (Table I).

There were no significant differences in age, gender, body weight, concentrations of plasma lipids, glucose, or creatinine (Table II), calculated $\mathrm{BMI}$ or the incidence of diabetes or smoking (data not included in Table II) between the five randomly selected therapeutic subgroups at the initial visit. Subgroups randomly assigned to treatment with quinapril, amlodipine, hydrochlorothiazide, losartan and bisoprolol did not differ before treatment with respect to blood pressure, carotid-femoral PWV values, concentrations of metalloproteinases or TIMP-1 (Table II). During the 6-month observation period, patients were not taking any other medications besides the ones being studied. At the final visit, there was no significant difference in BMI, plasma lipids, or glucose and creatinine levels between the therapeutic subgroups. The heart rate was significantly lower only in the group treated with bisoprolol. Before treatment, in the study group, multiple regression analysis for the independent variable carotid-femoral PWV was performed. Blood pressure, age, gender, body mass index plasma concentrations of investigated 
Table I. Comparison between study and control group

\begin{tabular}{|c|c|c|c|c|c|}
\hline \multirow[t]{2}{*}{ Variable } & \multicolumn{3}{|c|}{ Baseline } & \multicolumn{2}{|c|}{ After 6-month follow-up } \\
\hline & $\begin{array}{l}\text { Study group } \\
\qquad(N=95)\end{array}$ & $\begin{array}{l}\text { Control group } \\
\qquad(N=31)\end{array}$ & $P$-value & $\begin{array}{l}\text { Study group } \\
\qquad(N=95)\end{array}$ & $\begin{array}{l}\text { Control group } \\
\qquad(N=31)\end{array}$ \\
\hline Age [years] & $53.1(13.0)$ & $53.0(13.2)$ & 0.97 & $53.1(13.0)$ & $53.0(13.2)$ \\
\hline Male gender (\%) & $51(53.6)$ & $16(51.6)$ & 0.94 & $51(53.6)$ & $16(51.6)$ \\
\hline Body weight [kg] & $80.3(14.8)$ & $71.5(10.9)$ & 0.003 & $79.7(14.2)$ & $71.5(10.9)$ \\
\hline Height $[\mathrm{cm}]$ & $169.7(8.4)$ & $169.9(8.8)$ & 0.90 & & \\
\hline Obese patients, $n$ (\%) & $12(12.6)$ & $2(6.4)$ & 0.006 & $12(12.6)$ & $2(6.4)$ \\
\hline $\begin{array}{l}\text { Patients with diabetes type } 2 \text {, } \\
n(\%)\end{array}$ & $6(6.3)$ & $2(6.4)$ & 0.87 & $6(6.3)$ & $2(6.4)$ \\
\hline Smokers, $n(\%)$ & $12(12.6)$ & $4(12.9)$ & 0.56 & $12(12.6)$ & $4(12.9)$ \\
\hline $\mathrm{HR}[\mathrm{bpm}]$ & $73.1(10.8)$ & $72.1(7.4)$ & 0.62 & $69.6(12.3)$ & $70.2(7.7)$ \\
\hline $\mathrm{SBP}[\mathrm{mm} \mathrm{Hg}]$ & $153.5(19.4)$ & $131.1(14.2)$ & $<0.0001$ & $136.9(15.4)$ & $129.4(11.0)$ \\
\hline $\mathrm{DBP}[\mathrm{mm} \mathrm{Hg}]$ & $94.2(10.3)$ & $82.5(8.0)$ & $<0.0001$ & $85.1(9.3)$ & $80.8(8.5)$ \\
\hline $\mathrm{PWV}[\mathrm{m} / \mathrm{s}]$ & $10.6(1.6)$ & $9.8(1.2)$ & 0.011 & $10.0(1.7)$ & $9.7(1.4)$ \\
\hline Creatinine $[\mu \mathrm{mol} / \mathrm{l}]$ & $67.6(13.0)$ & $65.4(11.6)$ & 0.38 & $65.8(16.1)$ & $67.8(11.5)$ \\
\hline Glucose $[\mathrm{mmol} / \mathrm{l}]$ & $5.1(0.9)$ & $4.9(0.9)$ & 0.65 & $5.0(0.7)$ & $5.0(0.9)$ \\
\hline Total cholesterol [mmol/l] & $5.4(0.9)$ & $4.7(0.9)$ & 0.001 & $5.12(0.6)$ & $4.7(0.8)$ \\
\hline Triglycerides [mmol/l] & $1.5(0.7)$ & $1.3(0.7)$ & 0.18 & $1.66(0.7)$ & $1.46(0.6)$ \\
\hline $\mathrm{HDL}[\mathrm{mmol} / \mathrm{l}]$ & $1.4(0.4)$ & $1.5(0.5)$ & 0.21 & $1.41(0.4)$ & $1.58(0.4)$ \\
\hline LDL [mmol/l] & $3.3(0.8)$ & $2.6(0.9)$ & 0.001 & $2.97(0.6)$ & $2.59(0.7)$ \\
\hline
\end{tabular}

Data are given as mean (SD) or number (n) (percentage \%); HR - heart rate, SBP - office systolic blood pressure, DBP - office diastolic blood pressure, PWV - carotid-femoral pulse wave velocity, HDL - high-density lipoprotein, $L D L$ - low-density lipoprotein. $P$-value for the comparison between study and control group at the baseline. The differences ( $p$-values) between study and control group after a 6-month period did not differ from baseline $p$-values.

metalloproteinases as well as total cholesterol and LDL values were included in the regression model. Significant associations $\left(R^{2}=0.41, p=0.000003\right)$ of PWV with age $(B=0.408, p=0.00027)$, SBP $(B=0.441, p=0.0011)$ and serum concentrations of MMP-3 ( $B=0.204, p=0.0459)$ were found. In a regression analysis in the control group, based on the same model, PWV was related only to age $\left(R^{2}=0.22, B=0.471 ; p=0.011\right)$.

\section{Effect of antihypertensive therapy}

The analysis of variance (ANOVA) showed that, in the course of treatment in all therapeutic subgroups there was a significant reduction of systolic and diastolic blood pressure and carotid-femoral PWV. There were no significant differences in the magnitude of these effects between the subgroups (Table III).

The analysis of covariance (ANCOVA) adjusted for age, gender, the extent of the reduction of systolic blood pressure $(\triangle S B P)$ and the initial value of PWV did not show a significant difference in the magnitude of the decrease in PWV $(\triangle P W V)$ between the compared therapeutic subgroups (Figure 1).

After 6 months of treatment, regardless of the drug used, there was a significant decrease in plasma concentrations of MMP-2 $(p=0.039)$ and MMP-3 $(p=0.0001)$ and an increase in plasma concentration of TIMP-1 ( $p=0.0001)$ (Table IV). There were no significant changes in the concentrations of other metalloproteinases in all subgroups of the study group between the initial and final visit. To analyze the factors affecting the observed decrease in PWV $(\triangle \mathrm{PWV})$, multiple regression analysis was performed which included the following variables in the regression model: age, sex, body mass index, total cholesterol level, initial PWV, a reduction of the systolic blood pressure $(\triangle \mathrm{SBP})$, a decrease in MMP-3 concentration ( $\triangle M M P-3)$, a decrease in MMP-2 concentration ( $\triangle M M P-2)$, and an increase in TIMP-1 ( $\triangle$ TIMP-1) and type of medication. The reduction of PWV was significantly associated with a decrease in $\operatorname{SBP}(B=0.02, p=0.01)$, BMI $(B=0.06, p=0.028)$ and PWV value at the initial visit $(B=-0.37$, $p<0.001)$. 


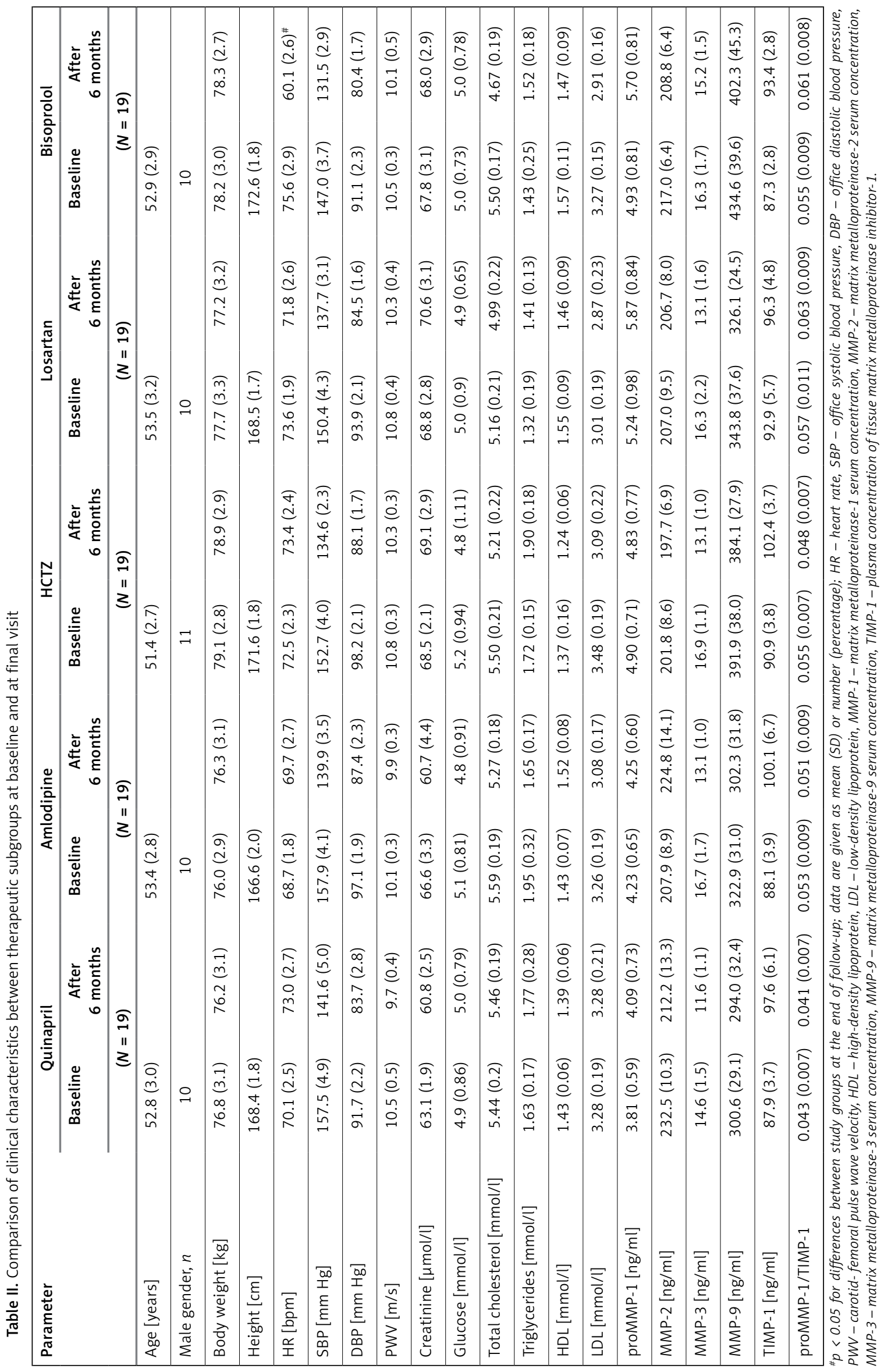




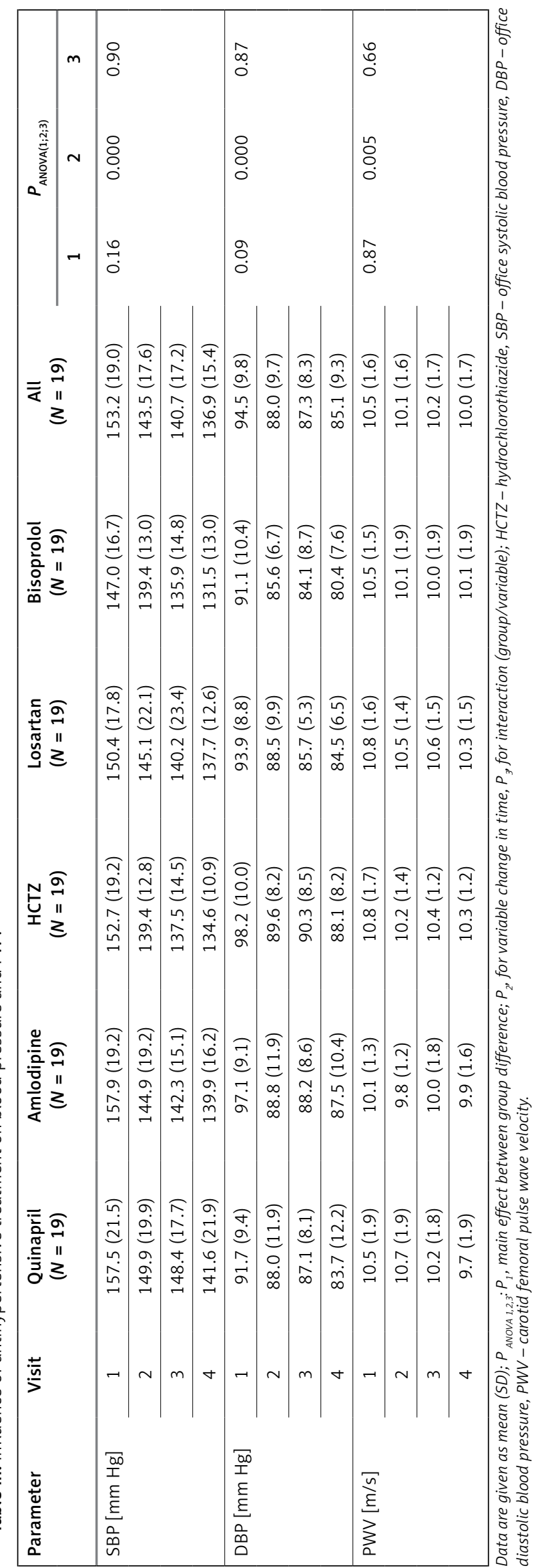

\section{Discussion}

The most important variable analyzed in our study, i.e. carotid-femoral PWV, was significantly higher in hypertensive patients than in the control group. In addition, the average value of PWV $(10.6 \mathrm{~m} / \mathrm{s})$ in the analyzed group of patients with hypertension significantly exceeded the average reference PWV value for the European population of healthy people aged $50-59$ years, which is $8.3 \mathrm{~m} / \mathrm{s}$ [38]. The majority of patients in our study group were within this age range.

Similarly to other studies, the PWV value in our hypertensive patients in the multiple regression analysis was significantly related to its typical determinants, i.e. age and systolic blood pressure [1, 12, 13, 38].

Therefore, this study-obtained association between PWV and the plasma concentration of MMP-3 (stromelysin-1) is the study novelty and has importance for better understanding of arterial stiffness mechanisms. In the control group of normotensive patients, the same analysis showed that the PWV value depended significantly on age only.

MMP-3 (stromelysin-1) plays an important regulatory role in the metabolism of connective tissue proteins. This enzyme initiates many processes by exposing the collagen and elastin fibers from a protective coating of proteoglycans and also activates the metalloproteinases responsible for their further degradation.

After 12 months of treatment with candesar$\tan$ in patients with arterial hypertension, Sasamura et al. [39] observed a significant decrease in the value of the arterial stiffness index ankle-brachial pulse wave velocity (abPWV), and an accompanying increase in the serum concentration of MMP-3. Moreover, the authors found a statisti-

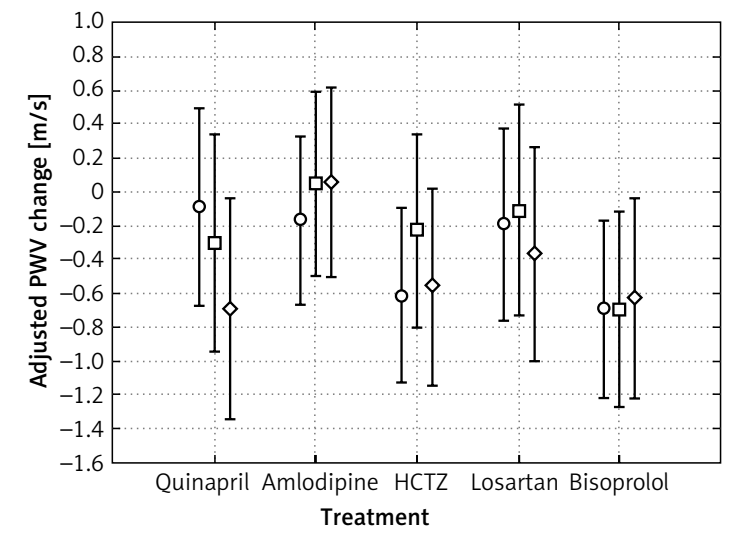

Figure 1. Changes from baseline to final visit in carotid-femoral pulse wave velocity (in patients randomized to therapeutic subgroups (ANCOVA analysis)). Adjusted for age, gender, baseline PWV, systolic blood pressure, and reduction in SBP changes from baseline in mean PWV at visit $2(\mathrm{O})$, $3(\square)$ and $4(\diamond)$. Error bars show SEM. $P=0.5179$ for between treatment group difference 
cally significant relationship between a decrease of abPWV ( $\triangle \mathrm{abPWV})$ and an increase of MMP-3 $(\triangle M M P-3)$.

In the same study, Sasamura et al. found that the baseline abPWV and the baseline MMP-3 values were not related; their result opposes our study observations. The different indices used for the evaluation of arterial stiffness may, to some extent, explain the observed differences.

The carotid-femoral PWV in our study may reflect changes in the aorta and large elastic arteries. The ankle-brachial PWV in the study by Sasamura et al. also depended on the pulse wave propagation in smaller arteries with a major contribution of the smooth muscle component. Another possible reason for the disparity is the different treatment period (6 months in our study and 12 months in the study by Sasamura et al.).

This may explain why the obtained results in regard to MMP-3 changes (an increase in Sasamura's study and a decrease in our study) during the treatment are conflicting.

The extent of the decrease in MMP-3 ( $\triangle M M P-3)$ and PWV ( $\triangle \mathrm{PWV}$ ) in our study was the same for all drug used, however these changes were not significantly associated, as it was demonstrated in Sasamura's study.

The extent of carotid-femoral PWV reduction observed in our study was not dependent on the antihypertensive drug used, but rather depended on the extent of the reduction of the systolic blood pressure. These results are contrary to the results of Karalliedde et al. [10], who obtained a significantly greater decrease in carotid-femoral PWV in patients treated with a valsartan/hydrochlorothiazide combination for 6 months, compared to treatment with amlodipine, with the same blood pressure lowering effect.

The independence of PWV lowering effect from the drug used, which makes a difference between our study and Karalliede study can be explained by a number of reasons: the use of valsartan/hydrochlorothiazide combination in one of the groups compared by Karalliedde et al. and monotherapy in all treatment subgroups in our study; the individual, selective, and more beneficial effect of valsartan than losartan on arterial stiffness; ethnic differences (i.e. compared groups were ethnically diverse - only $47 \%$ of subjects were of white origin in the study by Karalliedde et al. and they were 100\% white Caucasian in our study), different clinical characteristics (only patients with isolated systolic hypertension and type 2 diabetes with microalbuminuria were enrolled in the Karalliedde et al. study, while our study predominantly featured patients with mixed systolic-diastolic hypertension without diabetes (93.5\%)).

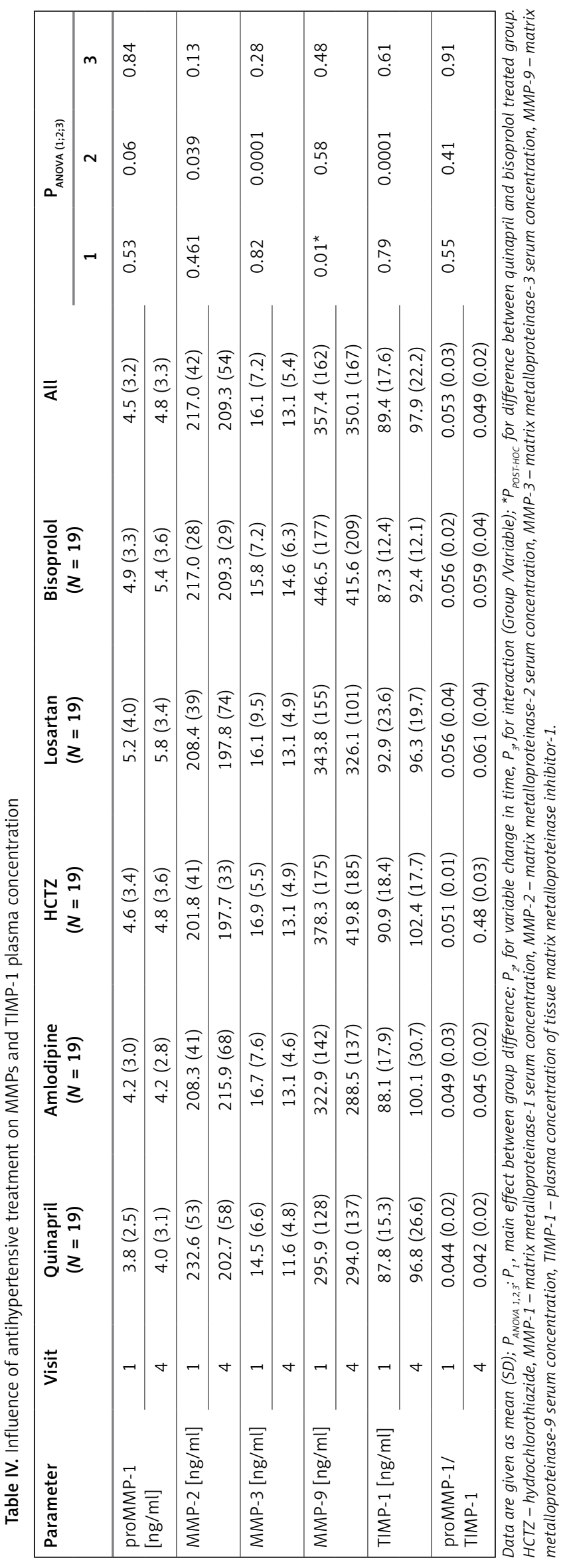


Differences in the clinical characteristics of the study groups also resulted in significantly higher PWV values (average of $12 \mathrm{~m} / \mathrm{s}$ ) in Karalliedde's group and lower values in our group of patients.

Therefore, this was potentially more difficult to reverse (not in the case of all drugs) in Karalliedde's group and easier in our group.

A meta-analysis conducted by Ong et al. [11] suggested that ACEI are more effective in reducing arterial stiffness, compared to other drugs in long-term treatment; however, our results do not support such a conclusion. For us, the meta-analysis by Ong is not overall conclusive in this issue, although it is cited by the latest ESH/ESC guidelines [3].

The number of patients in this meta-analysis in the long-term treatment $\operatorname{arm}(n=185)$ is less than twice the number of patients in our prospective study $(n=95)$. Of the nine studies in this meta-analysis, most recruited well below 19 patients in the active treatment group (one study had 28, and one had 17 patients). Each treatment subgroup of our study consisted of 19 patients. In most studies of the meta-analysis discussed, in the "long-term" treatment arm, the treatment period was only 1 month (although in one study it was 3 months and in another it was 6 months). Our results, which showed a lack of difference in the reduction of PWV for 5 studied drugs, are based on a 6-month treatment period. Therefore we believe our results are of great practical therapeutic relevance, until large-scale clinical trials provide more reliable evidence for the treatment guidelines in patients with systemic hypertension and arterial stiffness.

In the discussion of the results by Karalliedde et al. [10], they suggest that the superior effect in reducing arterial stiffness of the valsartan/hydrochlorothiazide combination compared to amlodipine is due to a specific effect on the activity of connective tissue matrix metalloproteinases.

For all metalloproteinases investigated by us, a significant treatment effect observed was a reduction of serum concentration of MMP-2 and MMP-3 and an increase in the concentration of TIMP-1. All the compared agents decreased the concentration of MMP-2 and MMP-3, and increased the concentration of TIMP-1 to the same extent, and the observed reduction of PWV was not dependent on changes in MMP-2, MMP-3 and TIMP-1 but was only dependent on a change in systolic blood pressure.

As in the study of Tan et al. [40] and Tayebjee et al. (Anglo-Scandinavian Cardiac Outcomes Trial ASCOT study) [31], and in contrast to the study of Li-Saw-Hee et al. [32] and Zervoudaki et al. [41], hypertensive patients in our study had higher levels of MMP-9 gelatinase than the control group.
Furthermore, in patients with hypertension we observed higher serum concentrations of another gelatinase, MMP-2 and a decrease in its concentration due to antihypertensive treatment. Zervoudaki et al. [41] observed a lower plasma concentration of MMP-2 in patients with arterial hypertension than in normotensives and an increase in levels of both gelatinases, MMP-2 and MMP-9, as a result of antihypertensive treatment. The aforementioned gelatinases have proteolytic activity against isolated collagen fibers and gelatin. Their elevated levels in the serum accompany inflammation and the destabilization of atherosclerotic plaque. The level of MMP-9 in the ASCOT study correlated with higher risk of coronary heart disease [31], and in patients with coronary artery disease it was proven to be a predictor of cardiovascular mortality [42]. Elevated levels of MMP-2 and MMP-9 in the serum of patients with hypertension and decreasing concentrations of these gelatinases in the course of treatment appears to be the most likely situation, as observed in our study.

A meta-analysis published in 2012 dedicated to the significance of metalloproteinases in heart and vascular remodeling in patients with arterial hypertension confirms that levels of MMP-9 are elevated in hypertensive patients without heart failure, compared to normotonics. Additionally, elevated levels of MMP-2 were observed in hypertensive patients and left ventricular diastolic failure, compared to patients with hypertension without diastolic failure [30]. The meta-analysis cited above also indicates the presence of elevated levels of TIMP-1 in patients with arterial hypertension compared to normotonics and suggests in the final conclusion that MMP-2, MMP-9 and TIMP-1 may play a role as biomarkers of cardiovascular remodeling in hypertension.

The significance of TIMP-1 in the metabolism of structural proteins of the vascular wall is probably greater than that of any separately considered metalloproteinase, with the exception of MMP-3. The broad spectrum of TIMP-1 activities includes blocking activity of MMP-1, inhibition of other metalloproteinases, and involvement in the processes of angiogenesis, apoptosis and steroidogenesis [18]. TIMP-1 is sometimes considered a marker of fibrosis and, therefore, could be involved in the development of arterial stiffness. Lindsay et al. [43] described its role in the development of fibrosis and myocardial stiffness. TIMP-1 lowers the turnover of type I collagen and probably increases the deposition of it in the arterial wall $[43,44]$, which could cause an increase in arterial stiffness.

Our results, however, do not support this hypothesis. In patients with hypertension, the plas- 
ma levels of TIMP-1 were not higher than those in the control group, as in the study by Stakos et al. [45]. Plasma concentrations of TIMP-1 showed no significant correlation with the PWV value as it was observed by Tayebjee et al. in the ASCOT study [31], where there was an increase of TIMP-1 rather than a decrease in the course of antihypertensive treatment. The results of Tayebjee et al. [31], both in terms of sample size and observation time during treatment (3 years), seem to be the most reliable. Limitations of our study include a small sample size, although it was similar to the majority of studies in which connective tissue matrix metalloproteinases were assessed. The second limitation of our study is the time of observation. We assumed 6 months of treatment as optimal for changes in PWV based on our previous experience. It is longer than in most of the studies that have assessed the effect of treatment on the change of PWV. Given the large heterogeneity of groups and discrepancies in the results of other studies that have evaluated the activity of metalloproteinases in patients with arterial hypertension, it is difficult to clearly determine whether the period of 6 months is optimal for the assessment of changes caused by treatment.

In conclusion, in patients with arterial hypertension, beside age and systolic blood pressure, the determinants of arterial stiffness include serum MMP-3 concentration. For drugs compared in the study with the same hypotensive effect obtained, the arterial stiffness reduction effect is not dependent on the drug used. Systolic blood pressure is one of the independent factors responsible for the reduction of arterial stiffness in the course of antihypertensive treatment.

\section{Acknowledgments}

This study was supported by the Polish Ministry of Science and Higher Education, grant no. NN402367333.

\section{Conflict of interest}

The authors declare no conflict of interest.

\section{References}

1. Laurent S, Cockcroft J, Van Bortel L, et al. Expert consensus document on arterial stiffness: methodological issues and clinical applications. Eur Heart J 2006; 27: 2588-605.

2. Laurent S, Boutourie P, Asmar R, Amar L. Aortic stiffness is an independent predictor of all cause mortality in hypertensive patients. Hypertension 2001; 37: 1236-41.

3. Mancia G, Fagard R, Narkiewicz K, et al. 2013 ESH/ESC guidelines for the management of arterial hypertension. The Task Force for the management of arterial hypertension of the European Society of Hypertension (ESH) and of the European Society of Cardiology (ESC). J Hypertens 2013; 31: 1281-357.
4. Guerin AP, Blacher J, Pannier B, Marchais SJ, Safar ME, London GM. Impact of aortic stiffness attenuation on survival of patients in end-stage renal failure. Circulation 2001; 103: 987-92.

5. Mahmud A, Feely J. Aldosterone-to-renin ratio, arterial stiffness, and the response to aldosterone antagonism in essential hypertension. Am J Hypertens 2005; 18: 50-5.

6. White WB, Duprez D, St Hilaire R, et al. Effects on the selective aldosterone blocker eplerenone versus the calcium antagonist amlodipine in systolic hypertension. Hypertension 2003; 41: 1021-6.

7. Asmar R, London GM, O'Rourke M, Safar ME. Improvement in blood pressure, arterial stiffness and wave reflections with a very-low-dose perindopril/indapamide combination in hypertensive patients a comparison with atenolol. Hypertension 2001; 38: 922-9.

8. Rajzer M, Klocek M, Kawecka-Jaszcz K. Effect of amlodipine, quinapril, and losartan on pulse wave velocity and plasma collagen markers in patients with mild-to-moderate arterial hypertension. Am J Hypertens 2003; 16: 439-44.

9. Mitchell GF, Dunlap ME, Warnica W, et al. Long-term trandolapril treatment is associated with reduced aortic stiffness. The Prevention of Events with Angiotensin-Converting Enzyme (PEACE) inhibition hemodynamic substudy. Hypertension 2007; 49: 1271-7.

10. Karalliedde J, Smith A, DeAngelis L, et al. Valsartan improves arterial stiffness in type 2 diabetes independly of blood pressure lowering. Hypertension 2008; 51: 1617-23.

11. Ong KT, Delerme S, Pannier B, et al. Aortic stiffness is reduced beyond blood pressure lowering by shortterm and long-term antihypertensive treatment: a meta-analysis of individual data in 294 patients. J Hypertens 2011; 29: 1034-42.

12. Laurent S, Boutouyrie P, Lacolley P. Structural and genetic bases of arterial stiffness. Hypertension 2005; 45 : 1050-5.

13. Zieman SJ, Melenovsky V, Kass DA. Mechanisms, pathophysiology, and therapy of arterial stiffness. Arterioscler Thromb Vasc Biol 2005; 25: 932-43.

14. McNulty M, Mahmud A, Spiers P, Feely J. Collagen type-I degradation is related to arterial stiffness in hypertensive and normotensive subjects. J Hum Hypertens 2006; 20: 876-3.

15. Skalska A, Gąsowski J, Cwynar M, Grodzicki T. The relationship between pulse wave velocity and indexes of collagen synthesis in hypertensive patients, according to the level of systolic blood pressure. J Hum Hypertens 2005; 19: 731-5.

16. Jacob MP. Extracellular matrix remodeling and matrix metalloproteinases in the vascular wall during aging and in pathological conditions. Biomed Pharmacother 2003; 57: 195-202.

17. Mäki-Petäjä KM, Wilkinson IB. Inflammation and large arteries. Potential mechanisms for inflammationinduced arterial stiffness. Artery Res 2012; 6: 59-64.

18. Mannello F, Gazzanelli G. Tissue inhibitors of metalloproteinases and programmed cell death: conundrums, controversies and potential implications. Apoptosis 2001; 6: 479-82.

19. Pawlak K, Pawlak D, Myśliwiec M. Urokinase-type plasminogen activator and metalloproteinase- 2 are independently related to the carotid atherosclerosis in haemodialysis patients. Thromb Res 2008; 121: 543-8.

20. Fiotti N, Altamura N, Orlando C, et al. Metalloproteinases-2, -9 and TIMP-1 expression in stable and unstable coronary plaques undergoing PCl. Int J Cardiol 2008; 127: 350-7. 
21. Matsuyama A, Sakai N, Ishigami M, et al. Matrix metalloproteinases as novel disease markers in Takayasu arteritis. Circulation 2003; 108: 1469-73.

22. Gluba-Brzózka A, Michalska-Kasiczak M, Franczyk-Skóra B, Nocuń M, Banach M, Rysz J. Markers of increased cardiovascular risk in patients with chronic kidney disease. Lipids Health Disease 2014; 13: 135-44.

23. Rysz J, Banach M, Stolarek RA, et al. Serum matrix metalloproteinases MMP-2 and MMP-9 and metalloproteinase tissue inhibitors TIMP-1 and TIMP-2 in diabetic nephropathy. J Nephrol 2007; 20: 444-52.

24. Vitilianowa K, Georgieva J, Milanowa M, Tzonev S. Blood pressure control predicts plasma matrix metalloproteinase-9 in diabetes mellitus type II. Arch Med Sci 2015; 11: 85-91.

25. Godin D, Ivan E, Johnson C, Magid R, Galis ZS. Remodeling of carotid artery is associated with increased expression of matrix metalloproteinases in mouse blood flow cessation model. Circulation 2000; 102: 2861-6.

26. Longo GM, Xiong W, Greiner TC, Zhao Y, Fiotti N, Baxter BT. Matrix metalloproteinases 2 and 9 work in concert to produce aortic aneurysms. J Clin Invest 2002; 110: 625-32.

27. Yasmin, Wallace S, McEniery CM, Dakham Z, Wilkinson IB. Matrix metalloproteinase-9 (MMP-9), MMP-2, and serum elastase activity are associated with systolic hypertension and arterial stiffness. Arterioscler Thromb Vasc Biol 2005; 25: 372-8.

28. Kingwell BA, Medley TL, Waddell TK, Cole TJ, Dart AM, Jennings GI. Large artery stiffnes: structural and genetic aspects. Clin Exp Pharmacol Physiol 2001; 28: 1040-3.

29. Medley TL, Kingwell BA, Gatzka CD, Pillay P, Cole TJ. Matrix metalloproteinase-3 genotype contributes to age-related aortic stiffening through modulation of gene and protein expression. Circ Res 2003; 92: 1254-61.

30. Marchesi C, Dentali F, Nicolini E, et al. Plasma levels of matrix metalloproteinases and their inhibitors in hypertension: a systematic review and meta-analysis. J Hypertens 2012; 30: 3-16.

31. Tayebjee MH, Nadar S, Blann AD, Beevers G, MacFadyen RJ, Lip GYH. Matrix metalloproteinase-9 and tissue inhibitor of metalloproteinase-1 in hypertension and their relationship to cardiovascular risk and treatment a substudy of the Anglo-Scandinavian Cardiac Outcomes Trial (ASCOT). Am J Hypertens 2004; 17: 764-9.

32. Li-Saw-Hee FL, Edmunds E, Blann AD, Beevers G, Lip GYH. Matrix metalloproteinase-9 and tissue inhibitor metalloproteinase-1 levels in essential hypertension. Relationship to left ventricular mass and anti-hypertensive therapy. Int J Cardiol 2000; 75: 43-7.

33. Definition and diagnosis of diabetes mellitus and intermediate hyperglycemia. Report of a WHO/IDF Consultation. 1. Diabetes mellitus - diagnosis. 2. Diabetes mellitus - classification. 3. Hyperglycemia. 4. Glucose tolerance test. I - World Health Organization. II - International Diabetes Federation. World Health Organization 2006. Printed by the WHO Document Production Services, Geneva, Switzerland.

34. O’Brien E, Asmar R, Beilin L. Practice guidelines of the European Society of Hypertension for clinic, ambulatory and self blood pressure measurement. J Hypertens 2005; 23: 697-701.

35. El Assad M, Topouchian J, Asmar R. Evaluation of two devices for self measurement of blood pressure according to the international protocol: the Omron M5-I and the Omron 705IT. Blood Press Monit 2003; 8: 127-33.
36. Asmar R, Benetos A, Topouchian J, et al. Assessment of arterial distensibility by automatic pulse wave velocity measurement. Validation and clinical application studies. Hypertension 1995; 26: 485-90.

37. Van Bortel LM, Laurent S, Boutouyrie P, et al. Expert consensus document on the measurement of aortic stiffness in daily practice using carotid-femoral pulse wave velocity. J Hypertens 2012; 30: 445-8.

38. The Reference Values for Arterial Stiffness Collaboration. Determinants of pulse wave velocity in healthy people and in the presence of cardiovascular risk factors: 'establishing normal and reference values'. Eur Heart J 2010; 31: 2338-50.

39. Sasamura K, Kitamura Y, Nakamura M, Ryuzaki M, Saruta T. Effects of the angiotensin receptor blocker candesartan on arterial stiffness and markers of extracellular matrix metabolism in patient with essential hypertension. Clin Exp Hypert 2006; 28: 511-20.

40. Tan J, Hua Q, Xing X, Wen J, Liu R, Yang Z. Impact of the metalloproteinase-9/tissue inhibitor of metalloproteinase- 1 system on large arterial stiffness in patients with essential hypertension. Hypertens Res 2007; 30: 959-63.

41. Zervoudaki A, Economou E, Stefanadis C, et al. Plasma levels of active extracellular matrix metalloproteinases 2 and 9 in patients with essential hypertension before and after antihypertensive treatment. J Hum Hypertens 2003; 17: 119-24.

42. Blankenberg S, Rupprecht $\mathrm{H}$, Poirier O, et al. Atherogene Investigators. Plasma concentrations and genetic variation of matrix melalloproteinase 9 and prognosis of patients with cardiovascular disease. Circulation 2003; 107: 1579-85.

43. Lindsay MM, Maxwell P, Dunn FG. TIMP-1 a marker of left ventricular diastolic dysfunction and fibrosis in hypertension. Hypertension 2002; 40: 136-41.

44. Laviades C, Varo N, Fernandez J, et al. Abnormalities of the extracellular degradation of collagen type I in essential hypertension. Circulation 1998; 98: 535-40.

45. Stakos DA, Tziakas DN, Chalikias GH, Mitrousi K, Tsiagalou C, Boudoulas H. Associations between collagen synthesis and degradation and aortic function in arterial hypertension. Am J Hypertens 2010; 23: 488-94. 